\title{
Norm estimations, continuity, and compactness for Khatri-Rao products of Hilbert Space operators
}

\author{
Arnon Ploymukda, Pattrawut Chansangiam* \\ Department of Mathematics, Faculty of Science, King Mongkut's Institute of Technology Ladkrabang, Bangkok 10520, Thailand \\ * Corresponding author: pattrawut.ch@kmitl.ac.th
}

\section{Article history}

Submitted 25 October 2017

Revised 13 May 2018

Accepted 11 July 2018

Published Online 3 December 2018

\begin{abstract}
We provide estimations for the operator norm, the trace norm, and the Hilbert-Schmidt norm for KhatriRao products of Hilbert space operators. It follows that the Khatri-Rao product is continuous on norm ideals of compact operators equipped with the topologies induced by such norms. Moreover, if two operators are represented by block matrices in which each block is nonzero, then their Khatri-Rao product is compact if and only if both operators are compact. The Khatri-Rao product of two operators are trace-class (Hilbert-Schmidt class) if and only if each operator is trace-class (Hilbert-Schmidt class, respectively).
\end{abstract}

Keywords: Khatri-Rao product, compact operator, Schatten $p$-class operator, trace-class operator, Hilbert-Schmidt class operator.

\section{INTRODUCTION}

Matrices and operators are fundamental tools in mathematics and related fields from viewpoints of theory, computations, and applications. A variety of ways to multiply matrices has been investigated in the literature. For instance, the Kronecker product and the Khatri-Rao product. Denote by $M_{m, n}(\mathbb{C})$ the set of $m$-by- $n$ complex matrices and abbreviate $M_{n, n}(\mathbb{C})$ to $M_{n}(\mathbb{C})$. Recall that the Kronecker product of $A=\left[a_{i j}\right] \in M_{m, n}(\mathbb{C})$ and $B \in M_{p, q}(\mathbb{C})$ is given by

$$
A \hat{\otimes} B=\left[a_{i j} B\right]_{i j} .
$$

The notion of Kronecker product was generalized to the Khatri-Rao product as follows. Consider two complex matrices $A$ and $B$ partitioned so that their $(i, j)$ th block are given by $A_{i j}$ and $B_{i j}$ for each $i, j$ (the sizes of $A_{i j}$ and $B_{i j}$ may be different). Then the Khatri-Rao product [1] of $A$ and $B$ is defined by

$$
A * \hat{*} B=\left[A_{i j} \hat{\otimes} B_{i j}\right]_{i j} .
$$

When $A$ and $B$ are of only one block, their Khatri-Rao product is just their Kronecker product. See more information in [2-6] and references therein.

The notion of Kronecker product of matrices is extended to the tensor product of operators on a Hilbert space. Certain algebraic, order, and analytic properties of tensor product of operators have been established, see [7-9]. In [10-11], the authors study the notion of tensor product for operators to the Tracy-Singh product of operators. Recently in [12-13], the authors introduced the Khatri-Rao product and the
Khatri-Rao sum for operators acting on the direct sum of Hilbert spaces. This construction provides a natural extension for both the Khatri-Rao product/sum for matrices, and the tensor product/sum of operators (see Section 2 for details). Fundamental algebraic, order, and structure properties of the Khatri-Rao product were investigated in [12].

In this paper, we continue developing this theory by discussing analytic properties of Khatri-Rao products for bounded linear operators acting on a Hilbert space. Under the assumption that two operators are represented by block matrices whose each block is nonzero, we will show that their Khatri-Rao product is compact if and only if both factors are compact. We provide norm bounds for the operator-norm, and the Schatten $p$-norms for $p=1,2, \infty$. Then we show that the Khatri-Rao product is (jointly) continuous with respect to the topologies induced by such norms. The norm bounds imply that the Khatri-Rao product of two operators are trace-class (Hilbert-Schmidt class) if and only if each operator is trace-class (Hilbert-Schmidt class, respectively).

This paper is organized as follows. In Section 2, we explain the notion of Khatri-Rao product for operators. In Section 3, we establish analytic properties involving norm bounds, continuity, convergence, and compactness of the Khatri-Rao product of operators in the operatornorm topology. The last section discusses the same properties of the Khatri-Rao product on norm ideals of compact operators.

\section{PRELIMINARIES ON KHATRI-RAO PRODUCTS FOR OPERATORS}

Throughout this paper, let $\mathrm{H}, \mathrm{H}^{\prime}, \mathrm{K}$ and $\mathrm{K}^{\prime}$ be complex Hilbert spaces. When $\mathrm{X}$ and $\mathrm{Y}$ are Hilbert spaces, let $\mathrm{B}(\mathrm{X}, \mathrm{Y})$ stand for the Banach space of all bounded linear operators from $\mathrm{X}$ into $\mathrm{Y}$, equipped with the operator norm $\|\cdot\|_{\infty}$. We abbreviate $\mathrm{B}(\mathrm{X}, \mathrm{X})$ to $\mathrm{B}(\mathrm{X})$. 
Recall that the tensor product of $A \in \mathrm{B}\left(\mathrm{H}, \mathrm{H}^{\prime}\right)$ and $B \in \mathrm{B}\left(\mathrm{K}, \mathrm{K}^{\prime}\right)$ is a unique bounded linear operator $A \otimes B$ in $\mathrm{B}\left(\mathrm{H} \otimes \mathrm{K}, \mathrm{H}^{\prime} \otimes \mathrm{K}^{\prime}\right)$ such that

$$
(A \otimes B)(x \otimes y)=A x \otimes B y
$$

for all $x \in \mathrm{H}$ and $y \in \mathrm{K}$. The tensor product is bilinear and continuous with respect to the topology induced by the operator norm.

From now on, fix the following orthogonal decompositions:

$$
\mathrm{H}=\bigoplus_{j=1}^{n} \mathrm{H}_{j}, \quad \mathrm{H}^{\prime}=\bigoplus_{i=1}^{m} \mathrm{H}_{i}^{\prime}, \quad \mathrm{K}=\bigoplus_{j=1}^{n} \mathrm{~K}_{j}, \quad \mathrm{~K}^{\prime}=\bigoplus_{i=1}^{m} \mathrm{~K}_{i^{\prime}},
$$

where all $\mathrm{H}_{j}, \mathrm{H}_{i}^{\prime}, \mathrm{K}_{j}, \mathrm{~K}_{i}^{\prime}$ are Hilbert spaces. For each $j=1, \ldots, n$, let $\underset{\text { by }}{E_{j}}: \mathrm{H}_{j} \rightarrow \mathrm{H}$ and $F_{j}: \mathrm{K}_{j} \rightarrow \mathrm{K}$ be the canonical embeddings defined

$$
\begin{aligned}
& E_{j}: x \mapsto(0, \ldots, 0, x, 0, \ldots, 0)(x \text { is in the } j \text {-th position }) \\
& \left.F_{j}: y \mapsto(0, \ldots, 0, y, 0, \ldots, 0) \text { ( } y \text { is in the } j \text {-th position }\right)
\end{aligned}
$$

For each $i=1, \ldots, m$, let $P_{i}: \mathrm{H}^{\prime} \rightarrow \mathrm{H}_{i}^{\prime}$ and $Q_{i}: \mathrm{K}^{\prime} \rightarrow \mathrm{K}_{i}^{\prime}$ be the orthogonal projections. Hence any operator $A \in \mathrm{B}\left(\mathrm{H}, \mathrm{H}^{\prime}\right)$ and $B \in \mathrm{B}\left(\mathrm{K}, \mathrm{K}^{\prime}\right)$ can be represented uniquely as operator matrices

$$
A=\left[A_{i j}\right]_{i, j=1}^{m, n} \text { and } B=\left[B_{i j}\right]_{i, j=1}^{m, n}
$$

where $A_{i j}=P_{i} A E_{j} \in \mathrm{B}\left(\mathrm{H}_{j}, \mathrm{H}_{i}^{\prime}\right)$ and $B_{i j}=Q_{i} B F_{j} \in \mathrm{B}\left(\mathrm{K}_{j}, \mathrm{~K}_{i}^{\prime}\right)$ for each $i, j$. We can perform the addition, the scalar multiplication, the adjointation, and the usual multiplication of operator matrices in a similar way to those of matrices.

We define the Khatri-Rao product of $A$ and $B$ to be a bounded linear operator from $\bigoplus_{j=1}^{n} \mathrm{H}_{j} \otimes \mathrm{K}_{j}$ to $\bigoplus_{i=1}^{m} \mathrm{H}_{j} \otimes \mathrm{K}_{j}$ represented by an operator matrix

$$
A^{*} B=\left[A_{i j} \otimes B_{i j}\right]_{i, j=1}^{m, n} .
$$

When $m=n=1$, the Khatri-Rao product $A^{*} B$ is reduced to the tensor product $A \otimes B$.

Lemma 1 ([12]). The Khatri-Rao product of operators is bilinear. More precisely,

$$
\begin{aligned}
A^{*}(B+C) & =A^{*} B+A^{*} C, \\
(B+C)^{*} A & =B^{*} A+C^{*} A, \\
A^{*}(\alpha B) & =\alpha\left(A^{*} B\right)=(\alpha A)^{*} B
\end{aligned}
$$

for any compatible operators $A, B, C$ and for any scalar $\alpha$.

\section{NORM BOUNDS, CONTINUITY, CONVERGENCE AND COMPACTNESS OF KHATRI-RAO PRODUCTS IN THE OPERATOR-NORM TOPOLOGY}

In this section, we discuss norm estimation, continuity, convergence, and compactness of the Khatri-Rao product of operators with respect to the topology induced by the operator norm.

Recall the following bounds for the operator norm of operator matrices.
Lemma 2 ([14]). Let $A=\left[A_{i j}\right]_{i, j=1}^{n, n}$ be an operator matrix in $\mathrm{B}(\mathrm{H})$. Then

$$
n^{-2} \sum_{i, j=1}^{n}\left\|A_{i j}\right\|_{\infty}^{2}, \quad\|A\|_{\infty}^{2}, \quad \sum_{i, j=1}^{n}\left\|A_{i j}\right\|_{\infty}^{2} .
$$

The next theorem provides an upper estimate for the operator norm of Khatri-Rao product. Such bound depends on the number of blocks in the representation (2).

Theorem 3. For any operator matrices $A=\left[A_{i j}\right]_{i, j=1}^{n, n} \in \mathrm{B}(\mathrm{H})$ and $B=\left[B_{i j}\right]_{i, j=1}^{n, n} \in \mathrm{B}(\mathrm{K})$, we have

$$
\|A * B\|_{\infty} \quad, \quad n^{2}\|A\|_{\infty}\|B\|_{\infty} .
$$

Proof. Since each $(i, j)$-th block of $A^{*} B$ is given by $A_{i j} \otimes B_{i j}$, it follows from Lemma 2 and the Cauchy-Schwarz inequality that

$$
\begin{aligned}
\left\|A^{*} B\right\|_{\infty}^{2} & , \sum_{i, j}\left\|A_{i j} \otimes B_{i j}\right\|_{\infty}^{2} \\
& =\sum_{i, j}\left\|A_{i j}\right\|_{\infty}^{2}\left\|B_{i j}\right\|_{\infty}^{2} \\
& ”\left(\sum_{i, j}\left\|A_{i j}\right\|_{\infty}^{2}\right)\left(\sum_{i, j}\left\|B_{i j}\right\|_{\infty}^{2}\right) \\
& " n^{4}\|A\|_{\infty}^{2}\|B\|_{\infty}^{2} .
\end{aligned}
$$

Hence, we obtain the bound (5).

The next result asserts that the map $(A, B) \mapsto A^{*} B$ is (sequentially) continuous with respect to the operator-norm topology.

Theorem 4. Let $A=\left[A_{i j}\right]_{i, j=1}^{n, n} \in \mathrm{B}(\mathrm{H})$ and $B=\left[B_{i j}\right]_{i, j=1}^{n, n} \in \mathrm{B}(\mathrm{K})$ be operator matrices and let $\left(A_{r}\right)_{r=1}^{\infty}$ and $\left(B_{r}\right)_{r=1}^{\infty}$ be sequences in $\mathrm{B}(\mathrm{H})$ and $\mathrm{B}(\mathrm{K})$, respectively. If $A_{r} \rightarrow A$ and $B_{r} \rightarrow B$ (in operator norm), then $A_{r} * B_{r} \rightarrow A * B$.

Proof. Suppose that $A_{r} \rightarrow A$ and $B_{r} \rightarrow B$ in operator norm. By the continuity of the operator norm, we have $\left\|B_{r}\right\|_{\infty} \rightarrow\|B\|_{\infty}$ as $r \rightarrow \infty$. By using Lemma 1 and Theorem 3, we have

$$
\begin{aligned}
& \left\|A_{r} * B_{r}-A * B\right\|_{\infty} \\
& \text {, }\left\|\left(A_{r}-A\right) * B_{r}\right\|_{\infty}+\left\|A *\left(B_{r}-B\right)\right\|_{\infty} \\
& \text {, } n^{2}\left\|A_{r}-A\right\|_{\infty}\left\|B_{r}\right\|_{\infty}+n^{2}\|A\|_{\infty}\left\|B_{r}-B\right\|_{\infty} \\
& \rightarrow n^{2} \cdot 0 \cdot\|B\|_{\infty}+n^{2} \cdot\|A\|_{\infty} \cdot 0=0 \text {. }
\end{aligned}
$$

It follows that $A_{r} * B_{r} \rightarrow A * B$ in the operator-norm topology.

Next, we consider the compactness of the Khatri-Rao product of two operators. Recall that a linear operator $T: \mathrm{H} \rightarrow \mathrm{H}$ is said to be compact if and only if it can be written in the form

$$
T=\sum_{n=1}^{\infty} \lambda_{n}\left\langle x_{n}, \square y_{n}\right.
$$


where $\left\{x_{n}\right\}_{n \in \square}$ and $\left\{y_{n}\right\}_{n \in \square}$ are orthonormal sets in $\mathrm{H}$, and $\left(\lambda_{n}\right)_{n \in \square}$ is a sequence of positive real numbers with limit zero, called the singular values of $T$. The zero operator is an example of a compact operator. Every finite rank operator (between infinite/finite dimensional spaces) is compact. Every compact operator is always bounded and continuous with respect to the operator-norm topology.

Lemma 5 ([7]). Let $A=\left[A_{i j}\right] \in \mathrm{B}(\mathrm{H})$ and $B=\left[B_{i j}\right] \in \mathrm{B}(\mathrm{K})$ be operators such that $A_{i j}$ and $B_{i j}$ are nonzero for all $i, j$. Then the tensor product $A \otimes B$ is compact if and only if both $A$ and $B$ are compact.

Lemma 6 ([11]). Let $A=\left[A_{i j}\right] \in \mathrm{B}(\mathrm{H})$ be an operator matrix. Then $A$ is compact if and only if $A_{i j}$ is compact for all $i, j$.

The next result provides a necessary and sufficient condition for $A^{*} B$ to be compact.

Theorem 7. Let $A=\left[A_{i j}\right] \in \mathrm{B}(\mathrm{H})$ and $B=\left[B_{i j}\right] \in \mathrm{B}(\mathrm{K})$ be operators such that $A_{i j}$ and $B_{i j}$ are nonzero for all $i, j$. Then the KhtriRao product $A^{*} B$ is compact if and only if both $A$ and $B$ are compact.

Proof. First, suppose that $A$ and $B$ are compact. By Lemma 6, we deduce that $A_{i j}$ and $B_{i j}$ are compact for all $i, j$. It follows from Lemma 5 that $A_{i j} \otimes B_{i j}$ is compact for all $i, j$. Lemma 6 ensures the compactness of $A * B$. For necessity part, reverse the previous procedure.

\section{KHATRI-RAO PRODUCTS ON NORM IDEALS OF COMPACT OPERATORS}

In this section, we investigate the Khatri-Rao product on several norm ideals of $\mathrm{B}(\mathrm{H})$. Recall that any proper ideal of the algebra $\mathrm{B}(\mathrm{H})$ is contained in the ideal of compact operators. For any compact operator $A \in \mathrm{B}(\mathrm{H})$, let $\left(s_{i}(A)\right)_{i=1}^{\infty}$ be the sequence of decreasingly ordered singular values of $A$. For each $1, p<\infty$, the Schatten $p$ norm of $A$ is defined by

$$
\|A\|_{p}=\left(\sum_{i=1}^{\infty} s_{i}^{p}(A)\right)^{1 / p},
$$

or equivalently,

$$
\|A\|_{p}=\left(\operatorname{tr}|A|^{p}\right)^{1 / p}
$$

where $|A|^{p}$ is defined by the functional calculus. If $\|A\|_{p}$ is a nonnegative real number, then $A$ is called a Schatten $p$-class operator. The Schatten $\infty$-norm is just the operator norm. For each $1, p, \infty$, denote by $\mathrm{S}_{p}$ the Schatten $p$-class operators. In particular, $S_{1}$ and $S_{2}$ are known as the trace class and the HilbertSchmidt class, respectively. Each Schatten $p$-norm induces a norm ideal of $\mathrm{B}(\mathrm{H})$ and this ideal is closed under the topology generated by such norm.

In order to proceed, some auxiliary results are needed.

Lemma 8. ([14]). Let $A=\left[A_{i j}\right]_{i, j=1}^{n, n}$ be an operator matrix in $\mathrm{S}_{p}$.

(i) For $1, p, p, 2$, we have

$$
\sum_{i, j=1}^{n}\left\|A_{i j}\right\|_{p}^{2}, \quad\|A\|_{p}^{2}, \quad n^{4 / p-2} \sum_{i, j=1}^{n}\left\|A_{i j}\right\|_{p}^{2} .
$$

(ii) For $2, p, \infty$, we have

$$
n^{4 / p-2} \sum_{i, j=1}^{n}\left\|A_{i j}\right\|_{p}^{2}, \quad\|A\|_{p}^{2}, \quad, \quad \sum_{i, j=1}^{n}\left\|A_{i j}\right\|_{p}^{2} .
$$

Lemma 9. Let $1, p, \infty$. An operator matrix $A=\left[A_{i j}\right] \in \mathrm{B}(\mathrm{H})$ is a Schatten $p$-class operator if and only if $A_{i j}$ is a Schatten $p$-class operator for all $i, j$.

Proof. This is a direct consequence of the norm estimations in Lemma 8.

Lemma 10. Let $1, p, \infty$. Let $A=\left[A_{i j}\right]$ be an operator matrix in the class $\mathrm{S}_{p}$ and let $\left(A_{r}\right)_{r=1}^{\infty}$ be a sequence in $\mathrm{S}_{p}$ where $A_{r}=\left[A_{i j}^{(r)}\right]_{i, j=1}^{n, n}$ for each $r \in \square$. Then $A_{r} \rightarrow A$ in $\mathrm{S}_{p}$ if and only if $A_{i j}^{(r)} \rightarrow A_{i j}$ in $\mathrm{S}_{p}$ for all $i, j=1, \ldots, n$.

Proof. Lemma 9 assures that $A_{i j}$ and $A_{i j}^{(r)}$ belong to $\mathrm{S}_{p}$ for any $i, j=1, \ldots, n$ and $r \in \square$. Consider the case $1, p, 2$ and suppose that $A_{r} \rightarrow A$ in $\mathrm{S}_{p}$. For any fixed $i, j \in\{1, \ldots, n\}$, we have from the estimation (6) that

$$
\left\|A_{i j}^{(r)}-A_{i j}\right\|_{p}^{2}, \quad \sum_{i, j=1}^{n}\left\|A_{i j}^{(r)}-A_{i j}\right\|_{p}^{2}, \quad\left\|A_{r}-A\right\|_{p}^{2} .
$$

Hence, $A_{i j}^{(r)} \rightarrow A_{i j}$ in $\mathrm{S}_{p}$. Conversely, suppose that $A_{i j}^{(r)} \rightarrow A_{i j}$ in $\mathrm{S}_{p}$ for each $i, j$. Lemma 8 implies that

$$
\left\|A_{r}-A\right\|_{p}^{2}, \quad n^{4 / p-2} \sum_{i, j=1}^{n}\left\|A_{i j}^{(r)}-A_{i j}\right\|_{p}^{2} .
$$

Hence, $A_{r} \rightarrow A$ in $\mathrm{S}_{p}$.

Consider the case $2<p<\infty$ and suppose that $A_{r} \rightarrow A$ in $\mathrm{S}_{p}$ Using the norm estimations (7), we obtain

$$
\left\|A_{i j}^{(r)}-A_{i j}\right\|_{p}^{2}, \quad \sum_{i, j=1}^{n}\left\|A_{i j}^{(r)}-A_{i j}\right\|_{p}^{2}, \quad n^{2-4 / p}\left\|A_{r}-A\right\|_{p}^{2} .
$$

for any $i, j \in\{1, \ldots, n\}$. Since $A_{r} \rightarrow A$ in $\mathrm{S}_{p}$, we have $A_{i j}^{(r)} \rightarrow A_{i j}$ in $\mathrm{S}_{p}$. Conversely, suppose that $A_{i j}^{(r)} \rightarrow A_{i j}$ in $\mathrm{S}_{p}$ for each $i, j$. Applying Lemma 8, we get

$$
\left\|A_{r}-A\right\|_{p}^{2}, \quad \sum_{i, j=1}^{n}\left\|A_{i j}^{(r)}-A_{i j}\right\|_{p}^{2} .
$$

Hence, $A_{r} \rightarrow A$ in $\mathrm{S}_{p}$.

Consider the case $p=\infty$. Let $A_{r} \rightarrow A$ in $\mathrm{S}_{\infty}$. For any fixed $i, j \in\{1, \ldots, n\}$, we have from Lemma 2 that

$$
\left\|A_{i j}^{(r)}-A_{i j}\right\|_{\infty}^{2}, \quad \sum_{i, j=1}^{n}\left\|A_{i j}^{(r)}-A_{i j}\right\|_{\infty}^{2}, \quad n^{2}\left\|A_{r}-A\right\|_{\infty}^{2} .
$$


Hence, $A_{i j}^{(r)} \rightarrow A_{i j}$ in $\mathrm{S}_{\infty}$. Conversely, suppose that $A_{i j}^{(r)} \rightarrow A_{i j}$ in $\mathrm{S}_{\infty}$ for each $i, j$. The estimation (4) implies that

$$
\left\|A_{r}-A\right\|_{\infty}^{2}, \quad \sum_{i, j=1}^{n}\left\|A_{i j}^{(r)}-A_{i j}\right\|_{\infty}^{2}
$$

Thus $A_{r} \rightarrow A$ in $\mathrm{S}_{\infty}$.

The following result asserts that the Khatri-Rao product is continuous on the ideal of compact operators $S_{\infty}$.

Theorem 11. If a sequence $\left(A_{r}\right)_{r=1}^{\infty}$ converges to $A$ and a sequence $\left(B_{r}\right)_{r=1}^{\infty}$ converges to $B$ in $\mathrm{S}_{\infty}$, then $A_{r} * B_{r}$ converges to $A^{*} B$ in $\mathrm{S}_{\infty}$.

Proof. It follows from Theorem 4, Theorem 7, and the fact that $S_{\infty}$ is a closed set.

The following theorem supplies an upper bound for the Schatten 1norm of the Khatri-Rao product of operators.

Theorem 12. Let $A \in \mathrm{B}(\mathrm{H})$ and $B=\left[B_{i j}\right]_{i, j=1}^{n, n} \in \mathrm{B}(\mathrm{K})$. If $A$ and $B$ are compact, then we have the following bound:

$$
\|A * B\|_{1}, \quad n\|A\|_{1}\|B\|_{1} .
$$

Hence, $A * B$ is trace-class if and only if both $A$ and $B$ are traceclass.

Proof. Suppose that $A_{i j}$ and $B_{i j}$ are nonzero operators for all $i, j$. Then the Khatri-Rao product $A^{*} B$ is also compact by Theorem 7. It follows from the norm bound (6) and the Cauchy-Schwarz inequality that

$$
\begin{aligned}
\|A * B\|_{1}^{2}, & n^{2} \sum_{i, j}\left\|A_{i j} \otimes B_{i j}\right\|_{1}^{2} \\
= & n^{2} \sum_{i, j}\left\|A_{i j}\right\|_{1}^{2}\left\|B_{i j}\right\|_{1}^{2} \\
& , \quad n^{2}\left(\sum_{i, j}\left\|A_{i j}\right\|_{1}^{2}\right)\left(\sum_{i, j}\left\|B_{i j}\right\|_{1}^{2}\right) \\
& \quad n^{2}\|A\|_{1}^{2}\|B\|_{1}^{2} .
\end{aligned}
$$

Hence, we obtain the bound (8). If there is a zero block of $A_{i j}$ or $B_{i j}$, then $A_{i j} \otimes B_{i j}=0$ is compact, and we can apply the above procedure.

Theorem 13. Let $A \in \mathrm{B}(\mathrm{H})$ and $B=\left[B_{i j}\right]_{i, j=1}^{n, n} \in \mathrm{B}(\mathrm{K})$. If $A$ and $B$ are compact, then

$$
\|A * B\|_{2}, \quad\|A\|_{2}\|B\|_{2}
$$

Hence, $A^{*} B$ is a Hilbert-Schmidt operator if and only if both $A$ and $B$ are Hilbert-Schmidt operators.

Proof. We may suppose that $A_{i j}$ and $B_{i j}$ are nonzero for all $i, j$. Then the operator $A^{*} B$ is compact by Theorem 7. Then by Lemma 8(ii) and the Cauchy-Schwarz inequality, we have

$$
\begin{aligned}
\left\|A^{*} B\right\|_{2}^{2} & =\sum_{i, j}\left\|A_{i j} \otimes B_{i j}\right\|_{2}^{2} \\
& =\sum_{i, j}\left\|A_{i j}\right\|_{2}^{2}\left\|B_{i j}\right\|_{2}^{2}
\end{aligned}
$$

$$
\begin{aligned}
& , \quad\left(\sum_{i, j}\left\|A_{i j}\right\|_{2}^{2}\right)\left(\sum_{i, j}\left\|B_{i j}\right\|_{2}^{2}\right) \\
& , \quad\|A\|_{2}^{2}\|B\|_{2}^{2} .
\end{aligned}
$$

Hence, we obtain the bound (9).

The final result states that the Khatri-Rao product is sequentially continuous on the norm ideal of trace-class operators and the norm ideal of Hilbert-Schmidt class operators.

Theorem 14. Let $p \in\{1,2\}$. If a sequence $\left(A_{r}\right)_{r=1}^{\infty}$ converges to $A$ and a sequence $\left(B_{r}\right)_{r=1}^{\infty}$ converges to $B$ in the norm ideal $\mathrm{S}_{p}$, then $A_{r} * B_{r}$ converges to $A * B$ in $\mathrm{S}_{p}$.

Proof. Suppose that the sequences $\left(A_{r}\right)_{r=1}^{\infty}$ and $\left(B_{r}\right)_{r=1}^{\infty}$ converge to $A$ and $B$, respectively, in $\mathrm{S}_{1}$. By applying Lemma 1 and the bound (8), we get

$$
\begin{aligned}
\left\|A_{r} * B_{r}-A * B\right\|_{1} & =\left\|A_{r} * B_{r}-A * B_{r}+A * B_{r}-A * B\right\|_{1} \\
& , \quad\left\|\left(A_{r}-A\right) * B_{r}\right\|_{1}+\left\|A *\left(B_{r}-B\right)\right\|_{1} \\
& ” n\left\|\left(A_{r}-A\right)\right\|_{1}\left\|B_{r}\right\|_{1}+n\|A\|_{1}\left\|\left(B_{r}-B\right)\right\|_{1} \\
& \rightarrow 0 \cdot n\|B\|_{2}+n\|A\|_{2} \cdot 0=0 .
\end{aligned}
$$

Hence, $A_{r} * B_{r} \rightarrow A * B$ in $\mathrm{S}_{1}$.

Let the sequences $\left(A_{r}\right)_{r=1}^{\infty}$ and $\left(B_{r}\right)_{r=1}^{\infty}$ converge to $A$ and $B$, respectively, in $\mathrm{S}_{2}$. By using Lemma 1 and the bound (9), we have

$$
\begin{aligned}
\left\|A_{r} * B_{r}-A * B\right\|_{2} & =\left\|A_{r} * B_{r}-A * B_{r}+A * B_{r}-A * B\right\|_{2} \\
& ,\left\|\left(A_{r}-A\right) * B_{r}\right\|_{2}+\left\|A *\left(B_{r}-B\right)\right\|_{2} \\
& ”\left\|\left(A_{r}-A\right)\right\|_{2}\left\|B_{r}\right\|_{2}+\|A\|_{2}\left\|\left(B_{r}-B\right)\right\|_{2} \\
& \rightarrow 0 \cdot\|B\|_{2}+\|A\|_{2} \cdot 0=0 .
\end{aligned}
$$

Hence, $A_{r} * B_{r} \rightarrow A * B$ in $\mathrm{S}_{2}$.

\section{CONCLUSION}

We have provided a necessary and sufficient condition for the Khatri-Rao product of operators to be compact. Indeed, for two operators in which every block is nonzero, their Khatri-Rao product is compact if and only if both factors are compact. We establish estimations for the operator norm, the trace norm, and the HilbertSchmidt norm for Khatri-Rao products of Hilbert space operators. The Khatri-Rao product is continuous with respect to the topologies induced by such norms. It follows that the Khatri-Rao product of two operators are trace-class (Hilbert-Schmidt class) if and only if each operator is trace-class (Hilbert-Schmidt class, respectively).

\section{ACKNOWLEDGEMENT}

This research was supported by King Monglus's Institute of Technology Ladkrabang Research Fund, grant number KREF046101.

\section{REFERENCES}

[1] Khatri, C.G. and Rao, C.R., Solutions to some functional equations and their applications to characterization of probability distributions, Sankhya, 30(2), 167-180, 1968.

[2] Al Zhour, Z.A. and Kilicman, A., Extension and generalization inequalities involving the Khatri-Rao product of several positive matrices, J. Inequal. Appl., Article ID 80878, 21 pages, 2006. 
[3] Civciv, H. and Taurkmen, R., On the bounds for norms of Khatri-Rao and Tracy-Singh products of Cauchy-Toeplitz matrices, Seluk J. Appl. Math., 6(2), 43-52, 2005.

[4] Liu, S., Contributions to Matrix Calculus and Applications in Econometrics, PhD dissertation, Institute of Actuarial Science and Econometrics, University of Amsterdam, Amsterdam, 1995.

[5] Rao, C.R. and Rao, M.B., Matrix Algebra and Its Applications to Statistics and Econometrics, World Scientific, 1998.

[6] Van Loan, C.F., The ubiquitous Kronecker product, J. Comp. Appl. Math., 123, 85-100, 2000.

[7] Kubrusly, C.S. and Vieira P.C.M., Convergence and decomposition for tensor products of Hilbert space operators, Oper. Matrices, 2(3), 407416, 2008.

[8] Schechter, M., On the spectra of operators on tensor products, J. Funct. Anal., 4(1), 95-99, 1969.

[9] Zanni, J. and Kubrsly, C.S., A note on compactness of tensor product, Acta Math. Univ. Comenian., 84(1), 59-62, 2015.

[10] Ploymukda, A. Chansangiam, P. and W. Lewkeeratiyutkul, Algebraic and order properties of Tracy-Singh products for operator matrices, $J$. Comput. Anal. Appl., 24(4), 656-664, 2018.

[11] Ploymukda, A. Chansangiam, P. and W. Lewkeeratiyutkul, Analytic properties of Tracy-Singh products for operator matrices, Journal of Computational Analysis and Applications, 24(4), 665-674, 2018.

[12] Ploymukda, A. and Chansangiam, P., Khatri-Rao products for operator matrices acting on the direct sum of Hilbert spaces, J. Math., 2016 (Article ID 8301709), 7 pages, 2016.

[13] Ploymukda, A. and Chansangiam, P., Operator Inequalities Involving Khatri-Rao Sums and Moore-Penrose Inverses, Mal. J. Fund. Appl. Sci., 13(4), 742-746, 2017.

[14] Bhatia, R. and Kittaneh, F., Norm inequalities for partitioned operators and an application, Math. Ann., 287(1), 719-726, 1990. 